\title{
Antisemitismus, Großstadtfeindlichkeit und reaktionäre Kapitalismuskritik in der deutschsprachigen Geographie vor 1945
}

\author{
B. Michel \\ Universität Erlangen-Nürnberg, Institut für Geographie, Wetterkreuz 15, 91058 Erlangen, Germany \\ Correspondence to: B. Michel (bmichel@geographie.uni-erlangen.de)
}

Received: 4 February 2014 - Revised: 15 April 2014 - Accepted: 28 April 2014 - Published: 8 October 2014

\begin{abstract}
Kurzfassung. In der Forschung zur Geschichte der Geographie besteht eine auffällige Leerstelle bezüglich antisemitischen Denkens vor 1945. In diesem Beitrag wird versucht die Rolle und Funktion antisemitischer Elemente in der deutschsprachigen Geographie des Landschaftsparadigmas vor 1945 nachzuzeichnen. Es wird die These vertreten, dass die antisemitische Figur des Judentums als einem raum- und bodenlosen Volk, nicht nur tief in das geographische Denken eingelagert ist, sondern mit der seit 1918 stark antimodernen und abstraktionsfeindlichen Ausrichtung der Disziplin das Judentum gerade auch als Personifikation für die verhassten Momente von Modernisierung, Liberalismus und Urbanität fungiert. Wenn antisemitisches Denken im Vergleich zu nationalistischen und eurozentrischen Momenten auf den ersten Blick relativ unsichtbar erscheint, so liegt eine Ursache für diese geringe Sichtbarkeit im geringen Interesse landschaftskundlicher Geographie an Stadtgeographie und Prozessen der gesellschaftlichen Modernisierung.
\end{abstract}

\section{Einleitung}

Die enge Verbindung der Geographie in Deutschland mit Diskurs und Politik des Nationalsozialismus, ihre Komplizenschaft mit der Planung für die eroberten Gebiete und ihre Beiträge zur Legitimierung von völkischem Rassismus und Vernichtungskrieg sind seit den 1980er Jahren umfangreich diskutiert worden. Von dem Wenigen, was in der Disziplin an historiographischen Arbeiten in den letzten 30 Jahren entstanden ist, befasst sich ein erheblicher Teil mit der Epoche zwischen 1918 und 1945 und damit mit Praxis und Vorläufern der Geographie im Nationalsozialismus. Eine Leerstelle in der Forschung besteht dabei jedoch bezüglich der Rolle von Antisemitismus, beziehungsweise es fällt eine merkwürdige Abwesenheit der Frage des Antisemitismus in der Geographie vor 1945 auf. Diesem soll sich im vorliegenden Beitrag angenähert werden.

Ziel dieses Artikels ist es, weder den Nachweis anzutreten, dass die Mehrheit der deutschsprachigen GeographInnen antisemitischem Denken anhingen, noch soll es darum gehen, zu untersuchen, wie der offizielle Antisemitismus des NSRegimes die universitäre Geographie in alltagspraktischer oder konzeptioneller Weise beeinflusste. Beides wären eigenständige Untersuchungen, die hier nicht zu leisten sind. Vielmehr liegt der Fokus dieses kurzen und gewiss recht holzschnittartigen Textes auf ersten Überlegungen zur Rolle und Funktion antisemitischer Elemente im geographischen Schreiben und Denken der 1920er und 1930er Jahre und insbesondere des geographischen Schreibens und Denkens im Rahmen des Landschaftsparadigmas. ${ }^{1}$ Das Interesse gilt hier also weniger der Frage, wie „der“ Antisemitismus über „die“ Geographie kam, als vielmehr der Frage ob und wie ein spezifischer ,geographischer“ Antisemitismus in Teilen der deutschsprachigen Geographie artikuliert und rationalisiert wurde.

Während rassistische, nationalistische oder expansionistische Momente der Geographie bis 1945 in der Forschung umfangreich diskutiert wurden (Böhm, 2008) und dabei ein

\footnotetext{
${ }^{1}$ Im Rahmen dieses Beitrags liegt der Fokus auf einer länderkundlichen bzw. landschaftskundlichen Geographie nach Hettner und damit auf Autoren, die insbesondere ab den 1920er Jahren sich in Gegnerschaft zu Hettner sahen, wie Schlüter, Banse, Passarge und große Teile der Schulgeographie.
} 
Schwerpunkt auf Arbeiten im Rahmen der Geopolitik gelegt wurde, so ist die Literatur zum Antisemitismus in der Geographie sehr überschaubar. Eine systematische Bearbeitung erfahren Antisemitismus und antisemitisches Denken der deutschen Geographie auch in den umfänglicheren Arbeiten zu dieser Zeit (Schultz, 1980; Rössler, 1990; Heske, 1988) kaum. Zwar widmet sich Horst Alfred Heinrich in seiner einschlägigen Arbeit über die „Politische Affinität zwischen geographischer Forschung und dem Faschismus im Spiegel der Fachzeitschriften" auf einigen Seiten der Frage des Antisemitismus in der Geographie, während die Gesamtarbeit den bisher umfassendsten Text zur inhaltlichen Nähe geographischen und nationalsozialistischen Denkens bis 1945 darstellt, bleibt dieses Kapitel jedoch relativ dünn (Heinrich, 1991). Auch in anderen ausführlichen Beiträgen wird der Frage, welche Rolle Antisemitismus für die Artikulation und Rationalisierung geographischen Denkens spielte, wenig Platz zugestanden. Und gewiss ist Antisemitismus in der deutschsprachigen Geographie dieser Zeit zumeist weniger sichtbar und präsent, als beispielsweise Nationalismus. Dieser Artikel befasst sich also mit einer doppelten Abwesenheit. Mit der Abwesenheit einer kritischen Forschung zu Antisemistismus in der Geographie und mit einer scheinbaren Seltenheit explizit antisemitischer Aussagen einer Disziplin, die sich seit den späten 1920er Jahren in erheblichem Maße durch eine ideologische Nähe zum Nationalsozialismus auszeichnete.

Neben einigen Arbeiten zur Rolle von Karl Haushofer und Elementen antisemitischen Denkens in dessen Geopolitik (Diner, 1984; Kost, 1998), haben sich eine Reihe von Arbeiten mit Siegfried Passarge beschäftigt, einem der sicherlich aggressivsten Antisemiten unter den prominenteren Geographen in Deutschland. Gerade die jüngeren Arbeiten stammen dabei aber nicht aus der Geographie, sondern entstanden im Rahmen der NS-Forschung in den Geschichts- und Kulturwissenschaften (Berg, 2012; Sandner, 1990; Schlör, 2005, S. 332ff; Kost, 1998). Auch in diesem Beitrag werden die Arbeiten von Passarge eine Rolle spielen. Es wird aber argumentiert, dass dieser nicht nur als radikaler Außenseiter der Geographie zu verstehen ist, sondern vielfach Argumente verdichtet und in eine oft durchaus unerträgliche Rhetorik gebracht hat, die in der Geographie mitten im Sagbarkeitsraum angesiedelt waren. Denn, wenngleich Passarge auch zeitgenössisch als radikaler Vertreter der Disziplin wahrgenommen worden ist und stark kritisiert wurde, so wurde doch kaum in Frage gestellt, dass er als ordentlicher Professor ein legitimer Vertreter der Disziplin war. Ebenso wie Ewald Banse, ,zog er nur , auf Flaschen', was in der seriösen Geographie in sublimierterer Form nicht minder lebendig war“" (Schultz, 1980, S. 273).

Dieser Beitrag möchte die Rolle antisemitischen Denkens vor dem Hintergrund und durch die Verbindung antimoderner und antiurbaner Positionen in der Disziplin diskutieren. Die These ist, dass der moderne Antisemitismus, den man nicht verwechseln darf mit deutlich älteren Formen der Ju- denfeindschaft und einem vormodernen Antisemitismus (Postone, 2005[2003], S. 137; Bergmann, 2011), eine wichtige Funktion als Scharnier der Geographie nach 1918 einnahm, da sich in ihm Großstadtfeindlichkeit, Antimodernismus und Abstraktionsfeindlichkeit des Landschaftsparadigmas verbinden ließen. Das bedeutet nicht, dass das länderkundliche Paradigma als ein genuin antisemitisches bezeichnet würde, aber doch als eines, das in einem bestimmten historischen Kontext ${ }^{2}$ für eine Reihe von Autoren die Möglichkeit bereitstellte, dieses mit zentralen Momenten des modernen Antisemitismus zusammenzuführen. Es bedeutet damit auch, dass Antisemitismus in der Geographie mehr war als einige antijüdische Ressentiments, die in politischen und persönlichen Auseinandersetzungen (etwa gegenüber Hettner oder Philippson) aktiviert werden konnten. Vielmehr wurde dieser in einer spezifischen Weise in und an geographisches Denken eingepasst. So hat Passarge explizit betont, dass sein Antisemitismus das Ergebnis empirischer und ,leidenschaftsloser" wissenschaftlicher Arbeit und Beobachtung sei (Passarge, 1929, S. 5), er sich also gegenüber dem von ihm abgelehnten älteren Judenhass als Vertreter eines sachlichen, rationalen und objektiven Antisemitismus begriffen hat (zur Emotionsgeschichte des modernen Antisemitismus: Jensen und Schüler-Springorum, 2013; Kauders, 2013).

\section{Antisemitismus in der deutschsprachigen Geographie}

Antisemitismus war ein wesentliches Element des Nationalsozialismus und der Wissenschaften während des Nationalsozialismus. Es wurde mehrfach darauf hingewiesen, dass für die deutsche Geographie das Jahr 1933 keinen Bruch darstellte, dass geographisches Denken der 1920er Jahre vielmehr sehr offen nationalsozialistische Ideen vertrat (Heinrich, 1991) und ,die Jahre des Nationalsozialismus [...] in der Geschichte des Faches keine Entgleisung, sondern eine Weiterentwicklung von Ideen der Weimarer Zeit" bedeuteten (Böhm, 2008, S. 382). Hard hat in einer Besprechung des genannten Buches von Heinrich darauf hingewiesen, dass sich der geographische Diskurs dieser Zeit durch die weitgehende Abwesenheit demokratischer und republikanischer Positionen auszeichnete (Hard, 1993). Mit der Verarbeitung der Niederlage des ersten Weltkriegs hat sich in der deutschsprachigen Geographie in erheblichem Maße ein antimodernes, pessimistisches und antidemokratisches Denken etabliert und insbesondere mit dem langsamen Hegemonieverlust von $\mathrm{Au}$ toren wie Hettner zum Ende der 1920er Jahre an Einfluss

\footnotetext{
${ }^{2}$ Es ließe sich vermutlich zeigen, dass Ansätze einer länderkundlichen Perspektive in der Geographie in anderen Ländern in dieser Zeit nicht eine vergleichbare Entwicklung vollzogen, dass es sich hier also um einen deutschen Sonderweg handelt. Für den Hinweis auf die Grenzen einer stark ,germane-centrist“ Ausrichtung dieses Beitrags bedanke ich mich bei einem/einer der anonymen GutachterInnen.
} 
gewonnen (Wardenga, 1995; Schultz, 1996). Trolls vielzitierter Rechenschaftsbericht zur Neugründung der Geographie in Deutschland nach 1945 (Troll, 1947), in der dieser der Geographie eine weitergehende Unberührtheit von nationalsozialistischem Denken und Handelns bescheinigt, ist zu einem erheblichen Teil ein Versuch der Geographie einen Persilschein auszustellen und verharmlost deren persönliche, politische und theoretische Verbundenheit zum Nationalsozialismus in erheblichem Maße (Böhm, 2003). Über Antisemitismus wird dort wie in ähnlichen Beiträgen zumeist ganz geschwiegen.

Die Frage dieses Aufsatzes ist die nach dem antisemitischen Denken in der Geographie vor 1945. Gerade wenn Antisemitismus nicht als eine von außen oktroyierte Ideologie begriffen werden soll, die der Geographie oder ,der Wissenschaft" fremd gegenübersteht, sondern als wichtiges Moment geographischen Denkens angesehen werden kann, bevor und jenseits dessen, dass er von staatlicher Seite erfordert und erzwungen wurde, so stellt sich die Frage, wie dieser in die Anlage geographischen Denkens eingebaut wurde, und was die Spezifik eben jenes geographischen Antisemitismus war. Daher wird gerade auch auf Literatur aus den Jahren vor 1933 geblickt werden. Dies dient nicht zuletzt auch dazu, sich gegen die Unterstellung Wirths zu versichern, ,einige Nazi-freundliche Sätze“ in wissenschaftlichen Arbeiten der „Nazizeit" würden in unstatthafter Weise genutzt um jemanden „schon als Nazi“ zu bezeichnen, wo es doch lediglich Opportunismus und Mitläufertum gewesen sei und überhaupt dies ,,von erst nach 1939 oder gar erst nach 1945 geborene[n] Leute[n]" auch gar nicht zu beurteilen sei (Wirth, 1995, S. 14). In ähnlicher Weise begründet auch Kost die Textauswahl in seinem Beitrag zum Antisemitismus in der Geographie, einem der wenigen Texte zur Geschichte der Geographie, deren primäres Thema Antisemitismus ist (Kost, 1998, S. 286). Aber während Kost Geopolitik und andere Ansätze der Geographie zusammendenkt respektive eine Untrennbarkeit proklamiert, um eine für die Geographie entlastende Aufspaltung zwischen wissenschaftlicher Geographie und ideologischer Geopolitik zu vermeiden, liegt in diesem Aufsatz der Fokus auf Beiträgen im Rahmen einer Geographie des Landschaftsparadigmas. Dies scheint sinnvoll, um nicht nur die Existenz antisemitischen Denkens in der deutschen Geographie zu verdeutlichen, sondern eben dessen Rolle und Verortung innerhalb des hegemonialen Paradigmas der Geographie.

\subsection{Antisemitische Publikationen in der deutschsprachigen Geographie}

Auf den ersten Blick scheint die Zahl geographischer Arbeiten, die sich mit Fragen nach Judentum und Juden befassen, relativ gering zu sein. Besonders vor dem ersten Weltkrieg findet eine Erwähnung meist in allgemeinen Beiträgen zu Palästina und Südosteuropa statt und ist dort Teil bevölkerungsgeographischer Darstellungen. Mit wenigen Aus- nahmen finden sich vor 1933 keine Monographien in der Geographie deren Titel eine klar antisemitische Stoßrichtung anzeigen würden. $\mathrm{Zu}$ diesen Ausnahmen gehören Bücher mit brachialen Titeln wie „Die Befreiung von der Judenfrage" des Wirtschaftsgeographen Ernst Tiessen (1922) oder Siegfried Passarges „Das Judentum als landschaftskundlichethnologisches Problem“ (1929). Auch in den zentralen Zeitschriften der Disziplin finden sich wenige explizit antisemitische Titel. Ein Umschwung und eine Hinwendung zur „Judenfrage“ setzen hier erst nach 1933 ein. Beispiele hierfür finden sich in Weigts Beitrag über „Die Verteilung der Juden im Deutschen Reiche“, Borgmans Beitrag zur Debatte um die Madagaskar-Frage „Palästina, Birobidjan oder Madagaskar? Eine geopolitische Untersuchung“ (Borgman, 1939) oder Bobeks ausführlicher Besprechung von Seraphims antisemitischen Werks „Das Judentum im osteuropäischen Raum“, das Bobek um eine räumliche Perspektive zu erweitern versucht (Bobek, 1939). Diese Beiträge von überwiegend jüngeren Autoren stehen zweifelsohne unter dem direkten Eindruck des Nationalsozialismus und den von diesem gestellten Anforderungen an eine Wissenschaft wie die Geographie und könnten daher im Sinne von Wirth als notwendiges Mitläufertum zu entschuldigen versucht werden.

Verglichen mit Expansionismus und Kolonialrevanchismus bleibt Antisemitismus in der Geographie insgesamt relativ unscheinbar. Dies mag auch angesichts der Konstruktion der Geographie als einer Wissenschaft des Raumes und in ihrer politisch-geographischen Ausrichtung des Raumhandelns der Völker und Staaten, eine zunächst auch wenig überraschende Tatsache sein.

Wird die These von der Bedeutung des Antisemitismus für die Geographie aufrechterhalten und dies auch für die Zeit vermutet, bevor dies durch den NS-Staat durchgesetzt wurde, so muss dies zunächst auf einer anderen Ebene betrachtet werden. Weniger in explizit antisemitischen Pamphleten und den expliziten „Vordenkern der Vernichtung“ (Aly und Heim, 1991), wie etwa dem überzeugten Nationalsozialisten Passarge. Vielmehr gilt es etwas kleinteiliger zu schauen.

Ein wichtiger Ausgangspunkt für eine Genealogie antisemistischen Denkens in der Geographie kann in der Neuformulierung der Disziplin zum Ende des 19. Jahrhunderts und der hier neuen Verschaltung von „Raum“, „Volk“ und einer vielfach darwinistisch oder lamarckianisch inspirierten Theorie (gesellschaftlicher) Entwicklung gesucht werden. So formuliert Friedrich Ratzel sowohl in seiner „AnthropoGeographie“ (Ratzel, 1882), wie in „Politische Geographie“ (Ratzel, 1897) die Vorstellung des Judentums als ein bodenund raumloses Volk und damit als eine Abweichung von der historischen Norm. Allerdings erscheint dieses in der überwiegenden Zahl in Form von Aufzählungen. Etwa als Beispiel für „landlose Völker“, die aufgrund dieser Eigenschaft nicht zum Interessensgebiet der politischen Geographie zählen: „die Juden, die schon in der römischen Kaiserzeit mehr in der Diaspora als in Judäa bedeuteten, die $\mathrm{Zi}$ geuner, die kleingewachsenen Jägervölker Innerafrikas, und 
zahllose ähnliche Existenzen, die ihre Stelle meist nicht so sehr in der politischen Geographie als in der politischen Ethnographie finden“ (Ratzel, 1897, S. 35). Es geht hier also nicht um eine dezidiert anti-jüdische Figur, als vielmehr um eine Figur nicht-sesshafter „Völker“ und „Rassen“, die aufgrund ihrer Nicht-Sesshaftigkeit und Raumlosigkeit nicht für die Politische Geographie interessant sind, sondern einer anderen Disziplin zugeordnet werden. An anderer Stelle ist von ,jüdischen, armenischen, griechischen, arabischen, betschuanischen u. a. Handelsrassen“ (Ratzel, 1882, S. 36) die Rede. Dies ist in beiden Fällen gewiss pejorativ, enthält antisemitische, antiziganistische und rassistische Elemente, bleibt aber auf einer anderen Ebene als die späteren Formulierungen, in denen diese negativen Konnotationen einerseits im Sinne eines modernen Antisemitismus mit einer absoluten Bedrohungserzählung und radikalen Handlungsaufforderung verbunden werden und andererseits zentrale Elemente der Konstitution des eigenen wissenschaftlichen Paradigmas berühren. Juden, Sinti und Roma oder nomadische Gesellschaften außerhalb Europas sind bei Ratzel zwar ein „Anderes“ des aus Perspektive eines deutschen Professors „normalen“ weltgeschichtlichen Entwicklungsprozesses, aber dies in einer wenig fundamentalen Weise. Die Spezifik des modernen Antisemitismus gegenüber anderen Formen ,gruppenbezogener Menschenfeindlichkeit" ist hier noch wenig expliziert. Durchaus ähnlich argumentiert Otto Maull in seiner in den frühen 1930er Jahren erschienen „Anthropogeographie“, die insgesamt schon deutlich stärker als Ratzel unter nationalsozialistischen Rassedenkens steht und der im Abschnitt zur Rassengeographie für eine Reinhaltung von Rassen (was für Ratzel kaum denkbar, noch wünschenswert gewesen wäre) argumentiert: „Bei der Gliederung der Menschheit [...] nehmen die Juden [...] und die Zigeuner, eine Sonderstellung ein. Beide entbehren eines geschlossenen Areals“ (Maull, 1932, S. 77). Die wesentlichen Momente und Spezifika des modernen Antisemitismus, der im letzten Drittel des 19. Jahrhunderts in Deutschland massiv an Einfluss gewann, erfüllen diese Darstellungen aber nur begrenzt. Für diesen modernen Antisemitismus sind die Vorstellung einer (verborgenen) jüdischen Macht, einer Dominanz von Jüdinnen und Juden im kapitalistischen Wirtschafts- und modernen Kulturleben sowie einer Bedrohung des traditionellen „Eigenen“ durch ein internationales Judentum konstitutiv (Postone, 2005[2003], S. 134ff; Holz, 2011). Dennoch liegen hier ganz wesentliche Grundlagen für die geographische Ausformulierung eines geographischen Antisemitismus, der genau diese Momente einschließt und folgenreich erweitert. Dafür war es aber nötig, dass die Geographie und ihre Paradigmen sich wandelten.

Eine Zunahme antisemitischer Publikationen in der Geographie lässt sich nach dem Ersten Weltkrieg feststellen. Diese nach 1918 einsetzende Zunahme an Beiträgen in denen antisemitisches Denken sichtbar wird, ist einmal mehr Beleg dafür, dass sich weite Teile der Geographie eben erst nach dem verlorenen Krieg zu einer in hohem Maße reaktionären, antiliberalen und antimodernen Wissenschaft entwickelten Böhm spricht vom „Versaille-Komplex“ einer ganzen Generation von Geographen (Böhm, 2003, S. 3), die zunehmend bemüht war, auch politische Forderungen im Dienste von Volk und Nation zu artikulieren (Schultz, 1996). Nicht nur der Verlust des Kriegs und die Suche nach einem für diese Niederlage Verantwortlichen, sondern eine ganze Reihe von Transformationserfahrungen und -prozessen, die sich etwa mit Harveys Begriff der Raum-Zeit-Kompression (Harvey, 1989) fassen lassen und die auf eine Erfahrung der Entzauberung der Welt, der Dynamisierung, Entankerung und wachsenden Differenzierung gesellschaftlicher Verhältnisse verweisen, tragen zu einer nachhaltigen Veränderung konservativen Denkens im frühen 20. Jahrhundert bei. Der Antimodernismus der Geographie muss damit als eine durch und durch moderne Denkfigur begriffen werden, als eine moderne Form der Krisenbewältigung, die durch den verlorenen Krieg und Prozesse der Modernisierung ausgelöst wurde. Er ist dieser Antimodernismus, eine moderne Konstruktion einer vormodernen Welt (Holz, 2011, S. 24). Dieser Antimodernismus findet sich auf sehr unterschiedlichen Ebenen: auf der Ebene geographischer Paradigmen, die mit ihrem Ganzheitlichkeits- und Unmittelbarkeitsversprechen dem Mainstream zeitgenössischer Wissenschafts- und Sozialtheorien vielfach zuwiderlaufen; auf der Ebene der Gegenstände geographischer Forschung, die in der Regel nicht Ausdruck gesellschaftlicher Modernisierungsprozesse sondern zumeist präindustrielle Raumproduktionen sind; bis hin zur Vorstellung des Geographen als einem ganzheitlichen Wissenschaftler, die starke Anleihen nimmt an romantischen Ideen und Subjektverständnissen (Eisel, 2008).

Und genau in diesem Rahmen stellt für die Geographie „das Judentum“ ein Problem dar, verkörpert es doch nicht nur eine Bedrohung der Beziehung von Volk und Raum beziehungsweise Volk und Landschaft, sondern steht es aufgrund einer im späten 19. Jahrhundert sich etablierenden Identifikation mit Moderne, Kapitalismus, Rationalismus und Internationalismus (Berg, 2011) doch zunehmend für das gesellschaftliche und politische Gegenmodell weiter Teile dieser Linie einer landschaftskundlichen Geographie. Die Bezeichnung der Juden als einem bodenlosen und „raumscheuen“ (Carl Schmitt zitiert nach: Berg, 2012, S. 6) Volk, einem Motiv, das einerseits die nun in der Geographie der 1920er Jahre organizistisch radikalisierte Idee homogener Völker im Raum in Frage stellte und das andererseits mit Mobilität und Wurzellosigkeit, das heißt insbesondere einem Mangel an „Gebundenheit an die Scholle“ im ländlichen Raum verbunden wird, erscheint nun als Bedrohung der eigenen nationalen, völkischen und auch wissenschaftlichen Identität (Kost, 2000, S. 180f). Gleichzeitig steht „,der Jude“ für den Inbegriff bzw. die Personifikation eben dieser Moderne und ihrer Probleme, wird dieser in geographischen Arbeiten seit den 1920er Jahren zunehmend mit jenen bekannten Momenten der Figur des internationalen Judentums identifiziert, zu denen sowohl der Bolschewismus wie das Finanzkapital, 
Erstarrung und Wurzellosigkeit, Pazifismus und Verweichlichung sowie Kriegstreiberei und Weltverschwörung zählen (Passarge, 1925, S. 136f).

So schreibt Passarge in „Das Judentum als landschaftskundlich-ethnologisches Problem“: „Der Bolschewismus ist nun ohne Zweifel jüdischer Entstehung. Juden sind seine Führer, Juden in Newyork haben ihn finanziert, Juden aus Newyork haben die russische Revolution durchgeführt, Juden in Rußland sie verbreitet und unterstützt.“ (Passarge, 1929, S. 15). Und, wie Heske gezeigt hat, setzt sich diese Argumentation gerade auch in der geographischen Lehre durch. So zitiert er ein zeitgenössisches Schulbuch: „Ebenso wie die Zigeuner sind die aus einer orientalischen Rassenmischung stammenden Juden uns vollkommen fremd. Schon in ihrer äußeren Gestalt und ihren Sitten prägt sich diese Wesensfremdheit aus ... Sie besitzen keinen eigenen Staat, haben sich aber in fast alle Länder Europas eingeschlichen. Durch ihre Gerissenheit und Rücksichtslosigkeit drängten sie sich in führende Stellungen der Wirtschaft und des Staates ein, wurden skrupellose Advokaten und vielfach gewissenlose Ärzte. Sie versuchten das Zeitungswesen und damit die öffentliche Meinung. Sie spielten eine beherrschende Rolle im Geldwesen. Als Politiker vergifteten sie das Staatsleben ihrer Wirtsvölker" (zitiert nach: Heske, 1988, S. 230).

Solche Identifizierungen von Juden mit als problematisch konstatierten Momenten der Moderne wie Urbanisierung und Finanzkapital, findet sich auch bei Autoren, die in der Geographie oftmals als NS-kritisch oder zumindest distanziert gelten. So ist für Troll in einem Aufsatz über den „Wandel der Volksordnung“ in Rumänien die Zunahme des ,Wuchers" identisch mit dem Anwachsen des Judentums (Troll, 1943, S. 50) und gibt es für Bobek ein ,unschöpferische[s] Wesen des jüdischen Städtertums“" (Bobek, 1939, S. 704). Dieses Denken gipfelt schon in den 1920er Jahren bei Passarge in einem exterminatorischen Antisemitismus und dem Vordenken der Vernichtung. So schreibt er in „Grundzüge der gesetzmäßigen Charakterentwicklung der Völker auf religiöser und naturwissenschaftlicher Grundlage und in Abhängigkeit von der Landschaft", einem Buch, dessen aggressiver Antisemitismus, Rassismus, Militarismus und brutalster Darwinismus kaum zu überbieten sind: „Es ist einfach das Gebot der Selbsterhaltung, das die Völker schließlich dazu zwingen wird, die jüdischen Gastarten wieder in die Gastkolonie zu verweisen. Die Lehren der Landschaftskunde, Rassenhygiene, der Charakterforschung und Kulturgeschichte vereinigen sich hier harmonisch zu der Vorstellung, daß es selbstmörderisch ist, sie in dem Staatskörper aufzunehmen; er geht zu Grunde, geradeso wie ein Körper schwer erkrankt, in dessen Blutkreislauf das Bacterium Coli gelangt“ (Passarge, 1925, S. 166). Und in folgender Abbildung aus dem als „leidenschaftslose" und rein wissenschaftliche Abhandlung angekündigten Buch „Das Judentum als landschaftskundlichethnologisches Problem“ (Passarge, 1929, S. 5) ist der Zivilisationsbruch der Shoa quasi graphisch antizipiert.

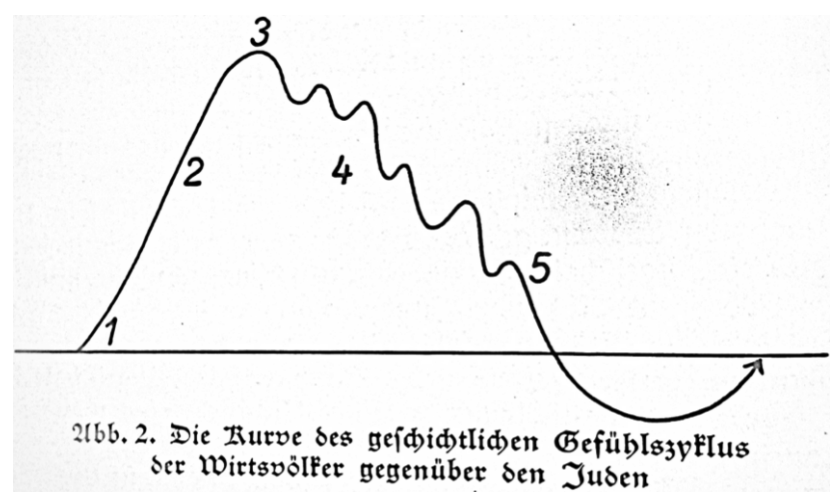

Abb. 1. „Die Kurve des geschichtlichen Gefühlszyklus der Wirtsvölker gegenüber den Juden“. Bezüglich Phase 5 verkündet der Text: „eine restlose Austreibung oder eine gründliche Unterdrückung der Juden.“ (Passarge, 1929, S. 24).

„Die Juden“ sind hier nun nicht mehr eine Gruppe unter vielen, sondern, so heißt es bevor einen Satz später die Begriffe Parasit und Wirtsvolk auftauchen, es spielt „,das Judentum ohne Zweifel eine besondere, und zwar für unser heutiges Kulturleben geradezu entscheidende Rolle" (Passarge, 1929, S. 20). Wenn Passarge zweifelsohne in vielerlei Hinsicht zu den radikalsten Antisemiten und aggressivsten Vertretern der Geographie in Deutschland zählt, der Geographen wie Philippson und Hettner antisemitisch denunzierte und der aktiv versuchte als liberal geltende Geographen zu diskreditieren und zu bekämpfen, so war die Grundfigur dieses Antisemitismus bei weitem nicht so außergewöhnlich und außerhalb des Raums des Sagbaren.

Juden werden nun zu einer Repräsentation respektive Personifikation eines wesentlichen Moments der Destabilisierung traditioneller Ordnungen - sei es betreffend des „Kulturlebens", der Siedlungsform oder der Ökonomie. Dies wird deutlicher, wenn man betrachtet, in welchen Kontexten sich antisemitisches Denken in der Geographie dieser Zeit artikuliert. Wird das Judentum vor dem ersten Weltkrieg primär als ein Thema ferner Länder diskutiert, so geht mit der allgemeinen Hinwendung zu einer stärker auf Deutschland fokussierenden Geographie nach 1918 einher, dass dieses nun hauptsächlich in Bezug auf seine räumliche Konkretion in der Großstadt in Erscheinung tritt. Und so mag die oben konstatierte Seltenheit explizit antisemitischer Texte in der deutschsprachigen Geographie eine Ursache darin haben, dass die Geographie den Großstädten gegenüber mit einem Gefühl zwischen Desinteresse und Feindschaft begegnete. Der Antisemitismus in der Geographie, so zeigt sich, ist eng verbunden mit stadtfeindlichen und insbesondere großstadtfeindlichen Motiven der Landschaftskunde. Bevor sich dem Verhältnis von Stadtkritik und Antisemitismus in der Geographie zugewandt werden kann, soll kurz diese Stadtfeindlichkeit der Geographie nach dem verlorenen Krieg skizziert werden. 


\subsection{Großstadtfeindschaft der länderkundlichen Geographie}

So wie Antimodernismus und ein moderner Antisemitismus erst in Folge des Ersten Weltkriegs zur dominierenden Ausrichtung der Geographie wurden, so gilt dies auch für die explizite Großstadtfeindlichkeit. Ratzel beispielsweise, bleibt bei der Frage nach Stellenwert und Bewertung stadtgeographischer Fragen ambivalenter (Ratzel, 1891, S. 496ff, 1903, 1906) als die gängige konservative Großstadtkritik des späten 19. und frühen 20. Jahrhunderts (z.B. Riehl, 1894; Spengler, 1922), für welche „der Steinkoloss“ der großen Städte „am Ende des Lebenslaufs einer jeden großen Kultur" steht (Spengler, 1922, S. 117). In dem von Karl Haushofer posthum herausgegebenen Sammelband „Erdenmacht und Völkerschicksal“ spricht Ratzel davon, dass die „Lebenskraft der Städte [...] viel größer als die der Landgebiete“ (Ratzel, 1940, S. 146) sei und auch seine Skizzen einzelner Städte zeigen kaum eine stadtkritische und kulturpessimistische Ausrichtung an. Ratzel steht denn auch 1939 im Auftakt zum vielzitierten stadtkritischen Buch „Mensch und Volk der Großstadt" des Politikers und Psychologen Willy Hellpach als wichtiger Vertreter einer nun im nationalsozialistischen Deutschland überkommenen Großstadtfreundlichkeit in der Geographie (Hellpach, 1939, S. 1). Allerdings war die deutschsprachige Geographie vor 1914 auch nicht sonderlich an Großstädten - dieser ,seltsamste[n] Schöpfung der Zivilisation“, wie Schrepfer noch Mitte der 1940er Jahre schrieb (Schrepfer 1944, S. 273) - interessiert. Und so finden sich in der Geographie keine Arbeiten die auf einer empirischen oder theoretischen Ebene vergleichbar wären mit einer zeitgenössischen Stadtsoziologie wie bei Simmel (1903), einer Theoretisierung ihrer Funktion in der Geschichte wie bei Weber (1972, S. 727-814) oder der späteren Chicago School (Park und Burgess, 1925). Das gilt auch für die oftmals als Begründung einer Stadtgeographie verstandenen Arbeiten von Otto Schlüter (1899).

Anders als die im Entstehen begriffene Soziologie, die ein wesentliches Moment ihrer Entstehung der Erfahrung der rapiden Urbanisierung der Gesellschaft zu verdanken hat, interessierte sich die Geographie, wie es sich insbesondere nach dem ersten Weltkrieg hegemonialisierte, nur wenig für große Städte und Fragen der Urbanisierung. Landschaftskunde war eben nur selten „Stadtlandschaftskunde“ (Passarge, 1930) und wenn sie es war, dann in der Regel vor dem Hintergrund eines deutlich stadtfeindlichen Duktus. Auch wenn Landschaft immer schon auch als Kulturlandschaft galt und die Geographie des Landschaftsparadigmas nie einem naiven Ideal unberührter Natur anhing, so waren es doch eher Äcker und kleine Siedlungen, als Industrieanlagen und Arbeitersiedlungen denen das Interesse galt - Arbeiten wie die von Hans Spethmann zum Ruhrgebiet sind hier eine Ausnahme, wurden aber eben auch als empirische und theoretische Ausnahmen wahrgenommen (Spethmann, 1928, 1938). In umfangreichen Länderkunden wurde in der Regel nur in sehr geringem Umfang auf Städte eingegangen. So räumt etwa Gradmanns zweibändiges „Süddeutschland“ von 1931 dem modernen München nur knapp zwei Seiten ein, allgemeine Überlegungen zu Städten reduzieren sich auf Fragen der historischen Stadtgründung (Gradmann, 1956[1931]). Auch der langsam sich durchsetzende Begriff der Stadtgeographie bezog sich in empirischen Arbeiten in der weit überwiegenden Zahl auf Klein- und Mittelstädte. Ideal einer Deutschen Stadt im Nationalsozialismus war der Geographie also weniger Speers Entwurf von Germania, als die zur „Nazi utopia“ gewandelte „deutscheste aller Städte“ Rothenburg ob der Tauber (Hagen, 2004). Der heute so dominante bias der geographischen Stadtforschung zugunsten von Großstädten ist abwesend. Und so stellte die 1933 von Friedrich Leyden ${ }^{3}$ veröffentlichte Monographie über „Gross-Berlin. Geographie der Weltstadt" (Leyden, 1933) sowohl aufgrund ihres Umfangs wie auch der weitgehenden Abwesenheit antiurbaner Einstellungen innerhalb der deutschsprachigen Geographie eine absolute Ausnahme dar. ${ }^{4}$ Gleichzeitig taucht „Die Stadt“ mit einer stärkeren Hinwendung zu geographischer Forschung in und zu Deutschland nach dem verlorenen Krieg stärker als ein geographisches Problem auf, als dies in der stark kolonialgeographisch geprägten Forschung zuvor.

Dem geographischen Diskurs des Landschaftsparadigmas wie auch der Geopolitik von Karl Haushofer nach 1918 bedeutete die Stadt und insbesondere die Großstadt ein nachhaltiger Verlust an Bodenständigkeit und Verwurzelung. Die mit ihr assoziierte Befreiung von den unmittelbaren Naturzwängen ließ die Stadt als naturfremd erscheinen. Die vom ländlichen Surplus ermöglichte Urbanisierung produzierte die Vorstellung von einem parasitären Charakter städtischen Lebens und die Vielfältigkeit städtischer Lebensweisen, die aus Migrationsbewegungen, Emanzipation und einem gesteigerten $\mathrm{Ma} ß$ an gesellschaftlicher Arbeitsteilung resultierte, ließ städtisches Leben als wurzellos und unstetig erscheinen. Werden Städte in positivem Licht geschildert, so vielfach gerade mit dem Hinweis darauf, dass diese es geschafft hätten, ,bodenständig“ zu bleiben und naturverbundene Ländlichkeit bewahrt zu haben (z.B. Hans Schrepfer in Bezug auf Würzburg: Schrepfer, 1967[1942], S. 217).

So wurde das Problem der Verstädterung, dem beispielsweise die Zeitschrift für Geopolitik 1933 mehrere Schwerpunktthemen widmete, in hohem Maße unter dem Titel der „Entwurzelung und Wurzellosigkeit des Großstadtmenschen“ diskutiert (Zeiss, 1933; Zeitschrift für Geopolitik, 1933). Aus geopolitischer Perspektive bedeuten Städte den

\footnotetext{
${ }^{3}$ Friedrich Leyden scheiterte Aufgrund seiner jüdischen Abstammung in den 1920 Jahren an einer Habilitation in der Geographie, arbeitete im Auswärtigen Amt und wurde 1933 aus dem Staatsdienst entlassen. Er wurde nach Theresienstadt deportiert und starb dort (Schlör, 2005, S. 320f).

${ }^{4}$ Bemerkenswert sind eventuell noch einige kürzere Dissertationen, die als „Stadtgeographie“ einzelner Städte angekündigt sind (Kröcher, 1913; Brüse, 1926; Müller, 1931; Schmidt, 1909)
} 
geographischen und bevölkerungswissenschaftlichen Autoren eine Gefahr der Entvölkerung und damit auch einer ,Entwehrung“ des Landes, auf deren Bevölkerungsnachschub die von sich aus nicht lebensfähigen Städte beständig angewiesen sind. „Die Großstadt ist, volksbiologisch gesehen, $\mathrm{Zu}$ schußbetrieb. Sie verschlingt nicht nur wie früher, den Bevölkerungsüberschuß des Landes, sondern greift - nachdem dieser Überschuß auf dem Lande immer geringer geworden ist - in die Substanz des Landvolkes ein und bedroht damit die wichtigste Grundlage der gesamten Volkskraft" (Burghöfer, 1941, S. 42). Zudem wird Verstädterung als Ursache des Geburtenrückgangs begriffen (Pfeil, 1933), dem sich nur durch Beharren auf traditioneller Lebensweise (Schmitthenner, 1930, S. 105) oder einer staatlichen Politik der aktiven „Verländlichung“ (Harmsen und von Loesch, 1933) widersetzt werden kann.

Mehr noch als einen Verlust eines wehrhaften Raumes durch die Konzentration der Menschen in Städten und den Verlust jeder Aussicht auf nationale Autarkie, stellt die Stadt den Ort problematischer Charakterentwicklung dar. Hatte schon Simmel (1903) dem Großstädter einen spezifischen Charakter, eben jenen viel zitierten intellektualistischen, blasierten und berechnenden großstädtischen Charakter zugeordnet und hierin einen zentralen Moment moderner Subjektivität im Kapitalismus festgemacht, so ist auch für diese großstadtfeindlichen Autoren die Stadt ein Ort eines großstädtischen Charakters. Dieser erhält aber eine deutlich pessimistischere Note. Der moderne Individualismus, der in der Großstadtkritik hier nicht als Folge moderner Rationalitäten, Produktionsweisen und Selbstverhältnisse, sondern als Folge der Stadt selbst sich herausbildet, wird als „Ichsucht“ pathologisiert. So schreibt Elisabeth Pfeil: „Individualistische und rationalistische Einstellung sind typische Merkmale des Stadtmenschen. Werden sie lebensfeindlich, dann haben wir den verstädterten Menschen“ (Pfeil, 1933, S. 171).

Ähnlich argumentiert Passarge, wenn er in „Die Deutsche Landschaft" schreibt: „Das Land erzeugt, die Stadt - vor allem die Groß- und Industriestadt - verbraucht Menschen und Familien. Das Land erzeugt brauchbare Charaktere, das Stadtleben verdirbt den Charakter." (Passarge, 1936, S. 97). Und so findet sich auch ein frappierendes Beispiel geographischer Großstadtfeindlichkeit im Kontext landschaftskundlichen Denkens bei Passarge und einer Reihe seiner Schüler. Zugrunde liegt diesem Passarges Versuch einer Typisierung von Charaktereigenschaften und deren kausale Abhängigkeit von Landschaftstypen - was ihm eine durchaus skurrile Debatte um die Frage nach dem Primat von Raum oder Rasse bringt, die er in Richtung eines Landschaftsdeterminismus auflöst, da beispielsweise auch die ,nordische Rasse in der Stadt genauso und vielleicht noch stärker" verfalle, als andere Rassen (Passarge, 1925, S. 4). In dem von Passarge herausgegebenen Sammelband ,Stadtlandschaften der Erde“ heißt es in einem Beitrag von Konrad Frenzel in vulgärdarwinistischer Weise, dass die Städte durch die in ihnen lokalisierte „Aufhebung des körperlichen Kampfes ums Da- sein“ nicht nur zu einer Verweichlichung und einem ungesunden Pazifismus führten, sondern dass in ihnen auch „,keine Auslese der natürlichen staatserhaltenden Fundamentalcharaktere mehr statt[finde ...]. Die staatserhaltenden Kardinaltugenden: persönlicher Mut, Ehrgefühl und physische Willenskraft, werden durch sartoide Eigenschaften ersetzt: Schlauheit, Gewandtheit, Gewissenlosigkeit" (Frenzel, 1930, S. 16). ${ }^{5}$

\section{Schluss: Antisemitismus und Großstadtfeindlichkeit}

Der neue geographische Antisemitismus der in der Geographie nach 1918 verstärkt auftaucht und sich dabei unterscheidet von Positionen vor 1914, ist eng verbunden mit Stadt und Urbanisierung und wird, das sollte deutlich geworden sein, in einer sehr ähnlichen Weise, mit ähnlichen Metaphern und ähnlichen Oppositionsbeziehungen als Problem artikuliert. Mit der Hinwendung zu Forschung über und in Deutschland rücken Urbanisierung, Industrialisierung und Moderne verstärkt in den Blick der Geographie. Allerdings in einer Weise, in der diese in zunehmendem Maße als problematisch und bedrohlich begriffen werden. Anschließend an eine ältere Diskurstradition in der europäischen Geistesgeschichte (Schlör, 2005), werden dabei Urbanität und Judentum miteinander verkoppelt. Die Stadt, so heißt es etwa in einem mehrfach veröffentlichten Text von Schrepfer, ist nicht nur der Ort an dem ,Naturverbundenheit, Naturinstinkt, unmittelbare Anschauung der Naturkräfte und Respekt und Ehrfurcht vor dem Walten der Natur [...] dem Menschen der Großstadt weitgehend verloren gegangen [sind]. In diesem Sinne ist in der Tat die Großstadt wurzellos." Dieses Motiv der Wurzellosigkeit der Großstadt und des entwurzelten Lebens in der Großstadt wird dabei zudem zum Ort wurzelloserer Charaktere. Die Stadt, so der Text weiter, wurde damit zum „Unterschlupf der Hefe des Volkes, Brutstätte asozialer Elemente, Hochburg des internationalen Judentums“" (Schrepfer, 1941, S. 243).

Das Judentum wird hier zur Personifikation einer entzauberten, kalkulierenden und rationalistischen modernen Gesellschaft ${ }^{6}$ beziehungsweise gerade deren Verwerfungen, „Dekandenz“ und Probleme. Hier kommen Antisemitismus und dessen Funktion für die konservative Kapitalismuskritik eines antimodernistischen Denkens ins Spiel, wird doch „die Gesellschaft gerade nicht als eine anonyme, apersonale, systemisch und strukturell verfasste Sozialform verstanden. Vielmehr wird sie ihrerseits personifiziert. Im Antisemitismus verkörpern die Juden die wurzellose, amoralische, durch Mittel wie Geld und Presse organisierte Gesellschaft, durch

\footnotetext{
${ }^{5} \mathrm{Zu}$ den Begriffen „Fundamentalcharakter“ und „Startoisierung" (Passarge, 1925). Ausführlicher hierzu die Arbeit von Joachim Schlör: (Schlör, 2005, S. 332ff).

${ }^{6}$ der Begriff der Gesellschaft ist insgesamt einer, der in der Geographie bis nach 1945 in erster Linie durch Abwesenheit auffällt.
} 
die sie die gemeinschaftliche Lebensform allmählich zersetzen und sich zu Weltherrschern aufschwingen." (Holz, 2011, S. 26) Jüdinnen und Juden werden damit nicht nur assoziiert mit großstädtischem Leben, sondern gelten zudem als Personifizierung des modernen Kapitalismus und der modernen Gesellschaft. Während etwa Georg Simmel die „Philosophie des Geldes“ (Simmel, 1989[1900]) und „Die Großstädte und das Geistesleben“ (Simmel, 1903) als zwei eng miteinander in Beziehung stehende Momente im Prozess der Moderne begriff, so werden der großstädtische Charakter und der abstrakte Kapitalismus über die Figur des Juden und der Jüdin personifiziert, sie werden zur Vergegenständlichung des Abstrakten. Strukturelle Momente der Moderne, des Kapitalismus, der Großstadt werden aber gerade darüber in konkrete Erscheinungen übersetzt und diese zu deren Wesen erklärt (Postone, 1982).

Einer Geographie, die sich als Wissenschaft der Verbindung von Mensch und Raum, Volk und Landschaft usw. begriff und politisch-militärische sowie moralische Vorbehalte gegen Großstädte, Modernität und Urbanität hegte, musste eine gesellschaftliche Gruppe, der Raumlosigkeit und Unstetigkeit sowie ein besonders großstädtischer Charakter zugeschrieben wurde, nicht nur suspekt sondern bedrohlich erscheinen. Ein Paradigma, dass die Geographie als eine Wissenschaft des Konkreten artikuliert und das als ein „Bollwerk gegen alle modernen Prozesse der Abstraktion“ (Eisel, 2008, S. 2) eingerichtet war, findet in jenen Menschen, denen ein Übermaß an Schlauheit und Gewandtheit statt Mut und Ehrgefühl zugeschrieben wird, die für abstrakten Tausch statt bodengebundener Arbeit, für raffendes und unschöpferisches statt schaffendes Handeln stehen und damit für die ,unfaßare[], zerstörerische[], unendlich mächtige[], internationale[] Herrschaft des Kapitals“ (Postone, 2005[1980], S. 190), sein Anderes. Dies ist für eine abstraktionsfeindliche Geographie, wie sie insbesondere, aber nicht allein, von Autoren wie Banse oder Passarge formuliert wurde, zwar wenig überraschend, es macht aber deutlich, dass antisemitische Motive und Strukturen viel tiefer in das geographische Denken eingelagert werden, also in abfällige Aussagen über das Judentum.

Edited by: B. Korf

Reviewed by: two anonymous referees

\section{Literatur}

Aly, G. and Heim, S.: Vordenker der Vernichtung. Auschwitz und die deutschen Pläne für eine neue europäische Ordnung, Hamburg: Hoffmann und Campe, 1991.

Berg, N.: Landschaftskunde und Wirtschaftsgeographie: Akademischer Antisemitismus im Werk Siegfried Passarges in den 1920er und 1930er Jahren, Flusser Studies, 14, 1-15, 2012.

Berg, Nicolas (Hrsg.): Kapitalismusdebatten um 1990. Über antisemitisierende Semantiken des Jüdischen, Leipzig: Leipziger Universitätsverlag, 2011.
Bergmann, W.: Geschichte des Antisemitismus, München: C.H. Beck, 2011.

Bobek, H.: Das Judentum im osteuropäischen Raum. Betrachtungen zu dem gleichnahmigen Werk von P. H. Seraphim, Deutsches Archiv für Landes- und Volksforschung, 3/4, 697-706, 1993.

Böhm, H.: Carl Troll (1899-1975) - Wissenschaftler in der NSZeit, in: Zeitumstände und Forschungsperspektiven, Herausgeber: Winiger, M., Carl Troll: Sankt Augustin: Ansgard, 1-99, 2003.

Böhm, H.: Geographie, in: Kulturwissenschaften und Nationalsozialismus, Herausgeber: Elvert, J. und Nielsen-Sikora, J., Stuttgart: Franz Steiner, 359-389, 2008.

Borgman, F.: Palästina, Birobidjan oder Madagaskar? Eine geopolitische Untersuchtung, Zeitschrift für Geopolitik, 16, 1-10, 1939.

Brüse, P.: Mühlheim a.d. Ruhr. Versuch einer Stadtgeographie, Köln (univ. Diss), 1926.

Burghöfer, F.: Die Großstädte im Lebensstrom des deutschen Volkes, in: Bevölkerungsbiologie der Großstadt, Herausgeber: von Eickstedt, E. F., Stuttgart: Ferdinand Enke, 41-55, 1941.

Diner, D.: „Grundbuch des Planeten“ Zur Geopolitik Karl Haushofers, Vierteljahrshefte für Zeitgeschichte, 32, 1-28, 1984.

Eisel, U.: Moderne Geographie mit atavistischen Methoden. Über die undeutliche Wahrnehmung eines deutlichen Paradigmas, in: Klassische Geographie. Geschlossenes Paradigma oder variabler Denkstil? Eine Kritik von Ulrich Eisel und eine Replik von HansDietrich Schultz, Herausgeber: Eisel, U. und Schultz, H.-D., Berlin: Geographisches Instut der Humboldt-Universität zu Berlin, 1-37, 2008.

Frenzel, K.: Die Deutsche Stadt im Mittelalter als Lebensraum, in: Stadtlandschaften der Erde, Herausgeber: Passarge, S., Hamburg: Friederichsen, de Gruyter \& Co., 15-28, 1930.

Gradmann, R.: Süddeutschland. Mit 49 Textabbildungen und 43 Tafeln und Karten, Darmstadt: Wissenschaftliche Buchgesellschaft, 1956 [1931].

Hagen, J.: The Most German of Towns: Creating an Ideal Nazi Community in Rothenburg ob der Tauber, Annals of the Association of American Geographers, 94, 207-227, 2004.

Hard, G.: Eine politisierte Disziplin, Geographische Zeitschrift, 81, 124-128, 1993.

Harmsen, H. and von Loesch, K.: Das bevölkerungspolitische Programm Mussolinis: „Entvölkerung der Städte“ und „Verländlichung“, Zeitschrift für Geopolitik, 123-125, 1933.

Harvey, D.: The Condition of Postmodernity - An Enquiry into the Origins of Cultural Change, Oxford: Basil Blackwell, 1989.

Heinrich, H.-A.: Politische Affinität zwischen geographischer Forschung und dem Faschismus im Spiegel der Fachzeitschriften, Giessen: Selbstverlag des Geographischen Instituts der Justus Liebig-Universität Giessen, 1991.

Hellpach, W.: Mensch und Volk der Großstadt, Stuttgart: Ferdinand Enke, 1939.

Heske, H.: ,... und morgen die ganze Welt ...“ Erdkundeunterricht im Nationalsozialismus, Giessen: Focus, 1988.

Holz, K.: Die Gegenwart des Antisemitismus, Islamistische, demokratische und antizionistische Judenfeindschaft, Hamburg: Hamburger Edition, 2011.

Jensen, U. and Schüler-Springorum, S.: Gefühle gegen Juden. Die Emotionsgeschichte des modernen Antisemitismus, Geschichte und Gesellschaft, 39, 413-442, 2013. 
Kauders, A.: Antisemitismus als Selbsthingebung, oder: Der Kampf gegen den ,jüdischen Rationalismus“, Geschichte und Gesellschaft, 39, 502-526, 2013.

Kost, K.: Anti-Semitism in German geography 1900-1945, Geojournal, 46, 285-291, 1998.

Kost, K.: Großstadtfeindlichkeit im Rahmen deutscher Geopolitik, in: Geopolitik. Grenzgänge im Zeitgeist, Herausgeber: Dieckmann, I., Krüger, P., und Schoeps, J. H.: Band 1.1. Potsdam: Verlag für Berlin - Brandenburg, 169-188, 2000.

Kröcher, H.: Stettin. Ein Beitrag zur modernen Stadtgeographei, Greifswald: Herrcke \& Uebling, 1913.

Leyden, F.: Berlin als Beispiel einer wurzellosen Großstadt, Zeitschrift für Geopolitik, 10, 176-188, 1933.

Maull, O.: Anthropogeographie, Berlin: Walter de Gruyter, 1932.

Müller, E.: Die Altstadt von Breslau. Citybildung und Physiognomie. Ein Beitrag zur Stadtgeographie, Breslau: M. \& H. Marcus, 1931.

Park, R. E. and Burgess, E. W. (Hrsg.): The City, Chicago, 1925.

Passarge, S.: Grundzüge der gesetzmäßigen Charakterentwicklung der Völker auf religiöser und naturwisenschaftlicher Grundlage und in Abhängigkeit von der Landschaft, Berlin: Gebrüder Borntraeger, 1925.

Passarge, S.: Das Judentum als landschaftskundlich-ethnologisches Problem, München: Lehmanns, 1929.

Passarge, S.: Die deutsche Landschaft, Berlin: Dietrich Reimer, 1936.

Passarge, Siegfried (Hrsg.): Stadtlandschaften der Erde, Hamburg: Friederichsen, de Gruyter \& Co., 1930.

Pfeil, E.: Zur Frage der Verädterung. Über den Zusammenhang von Verstädterung und Geburtenrückgang, Zeitschrift für Geopolitik, 10, 168-174, 1933.

Postone, M.: Nationalsozialismus und Antisemitismus. Ein theoretischer Versuch, Merkur, 1/1982, 13-25, 1982.

Postone, M.: Antisemitismus und Nationalsozialismus, in: Moishe Postone: Deutschland, die Linke und der Holocaust, Freiburg: ça ira, 165-194, 2005 [1980].

Postone, M.: Der Holocaust und der Verlauf des 20. Jahrhunderts, in: Moishe Postone: Deutschland, die Linke und der Holocaust, Freiburg: ça ira, 119-164, 2005 [2003].

Ratzel, F.: Anthropo-Geographie oder Grundzüge der Anwendung der Erdkunde auf die Geschichte, Stuttgart: J. Engelhorn, 1882.

Ratzel, F.: Antropogeographie. Zweiter Teil. Die geographische Verteilung des Menschen, Stuttgart: J. Engelhorn, 1891.

Ratzel, F.: Politische Geographie, München: R. Oldenbourg, 1897.

Ratzel, F.: Die geographische Lage der großen Städte, in: Jahrbuch der Gehe-Stiftung, Die Großstadt. Vorträge und Aufsätze zur Städteausstellung, Dresden: Jann \& Jaentsch, 33-72, 1903.

Ratzel, F.: Kleine Schriften. Ausgewählt und herausgegeben von Hans Helmolt. Band 1, München: R. Oldenbourg, 1906.

Ratzel, F.: Erdenmacht und Völkerschicksal. Eine Auswahl aus seinen Werken. Herausgegeben und eingeleitet von Generalmayor a.D. Prof. Dr. Karl Haushofer, Stuttgart: Alfred Kröner, 1940.

Riehl, W. H.: Land und Leute, Stuttgart: Cotta, 1894.

Rössler, M.: "Wissenschaft und Lebensraum". geographische Ostforschung im Nationalsozialismus. Ein Beitrag zur Disziplingeschichte der Geographie, Berlin: Reimer, 1990.

Sandner, G.: Zusammenhänge zwischen wissenschaftlichem Dissens, politischem Kontext und antisemitischen Tendenzen in der deutschen Geographie 1918-1945: Siegfried Passarge und Al- fred Philippson, in: Philippson - Gedächtnis - Kolloquium, Herausgeber: Ehlers, E., Bonn: Ferd. Dümmlers, 35-49, 1990.

Schlör, J.: Das Ich der Stadt. Debatten über Judentum und Urbanität, 1822-1938, Göttingen: Vandenhoeck \& Ruprecht, 2005.

Schlüter, O.: Bemerkungen zur Siedlungsgeographie, Geographische Zeitschrift, 5, 65-84, 1899.

Schmidt, H.: Citybildung und Bevölkerungs-Verteilung in Grossstädten. Ein Beitrag zur Entwicklungsgeschichte des modernen Städtewesens, München: Ernst Reinhardt, 1909.

Schmitthenner, H.: Die chinesische Stadt, in: Stadtlandschaften der Erde, Herausgeber: S. Passarge, Hamburg: Friederichsen, de Gruyter \& Co, 85-108, 1930.

Schrepfer, H.: Großstadtlandschaft und Großstadtmensch, Zeitschrift für Rassenkunde, 12, 230-243, 1941.

Schrepfer, H.: Zur Geographie der Großstadt und ihrer Bevölkerung, Zeitschrift für Erdkunde, 264-286, 1944.

Schrepfer, H.: Das Stadtbild von Würzburg, in: Allgemeine Geographie und Länderkunde, Ausgewählte Arbeiten zum Gedenken seines 70. Geburtstages am 21. Mai 1967, Herausgeber: Schrepfer, H., Wiesbaden: Franz Steiner, 199-219, 1967 [1942].

Schultz, H.-D.: Die deutschsprachige Geographie von 1800-1970. Ein Beitrag zur Geschichte ihrer Methodologie, Berlin: Selbstverlag des geographischen Instituts der Freien Universität Berlin, 1980.

Schultz, H.-D.: Die Geographie der Moderne: Eine Antimoderne Wissenschaft?, in: Der Weg der Deutschen Geographie, Herausgeber: Heinritz, G., 50. Deutscher Geographentag Potsdam 1995. Stuttgart: Steiner, S. 88-107, 1996.

Simmel, G.: Die Großstädte und das Geistesleben, in: Jahrbuch der Gehe-Stiftung (Hg.): Die Großstadt. Vorträge und Aufsätze zur Städteausstellung, Dresden: Jann \& Jaentsch, 185-206, 1903.

Simmel, G.: Philosophie des Geldes. Georg Simmel Gesamtausgabe Band 6, Frankfurt: Suhrkamp, 1989 [1900].

Spengler, O.: Der Untergang des Abendlandes. Umrisse eienr Morphologie der Weltgeschichte. Zweiter Band, Welthistoriche Perspektiven, München: C.H.Beck, 1922.

Spethmann, H.: Dynamische Länderkunde, Breslau: Ferdinand Hirt, 1928.

Spethmann, H.: Das Ruhrgebiet im Wechselspiel von Land und Leuten,Wirtschaft, Technik und Politik. Dritter band. Das Ruhrgebiet der Gegenwart, Berlin: Verlag für Sozialpolitik, Wirtschaft und Statistik, Paul Schmidt, 1938.

Tiessen, E.: Die Befreiung von der Judenfrage : ein Vorschlag. Der Anker: Flugschriften des Anker 8, Berlin: Universitas Buch und Kunst, 1922.

Troll, C.: Der Wandel der Volksordnung, Siedlung und bäuerlichen Wirtschaft im rumänischen Altreich, Zeitschrift der Gesellschaft für Erdkunde zu Berlin, 46-54, 1943.

Troll, C.: Die geographische Wissenschaft in Deutschland 1933 bis 1945, Erdkunde, 2, 3-48, 1947.

Wardenga, U.: Geographie als Chorologie. Zur Genese und Struktur von Alfred Hettners Konstrukt der Geographie, Stuttgart: Franz Steiner, 1995.

Weber, M.: Wirtschaft und Gesellschaft, Tübingen: Mohr Siebeck, 1972 [1922].

Wirth, E.: Einhundert Jahre Geographie in Erlangen. Eine Universitätsdisziplin im Kontext übergreifender wissenschaftlicher und hochschulpolitischer Zeitströmungen, Mitteilungen der Fränkischen Geographischen Gesellschaft, 42, 3-32, 1995. 
Zeiss, H.: Entwurzelund und Wurzellosigkeit. Beobachtungen und Fragen zum Problem der Verstädterung, Zeitschrift für Geopolitik, 10, 310-319, 1933.
Zeitschrift für Geopolitik (1933): Vom Absterben der Städte, Zeitschrift für Geopolitik, 10, 100-105, 1933. 